\title{
The Average Energy and Molar Specific Heat at Constant Volume of an Einstein Solid Measured by an Observer with Fluctuating Frame of Reference
}

\author{
Yun-Sok Shin \\ Sejong Academy of Science and Arts, Sejong, Republic of Korea \\ Email address: \\ yunsokshin@naver.com \\ To cite this article: \\ Yun-Sok Shin. The Average Energy and Molar Specific Heat at Constant Volume of an Einstein Solid Measured by an Observer with \\ Fluctuating Frame of Reference. American Journal of Physics and Applications. Vol. 7, No. 1, 2019, pp. 21-26. \\ doi: 10.11648/j.ajpa.20190701.14
}

Received: December 15, 2018; Accepted: January 11, 2019; Published: February 22, 2019

\begin{abstract}
We report an observer effect in an Einstein solid, a quantum-mechanical system, induced by fluctuations of an observer's frame of reference; which has been studied so far under the assumption that the observer's frame of reference remains constant throughout the performance of a measurement, thus, what is actually measured throughout the performance of a measurement is an unresolved problem during which the observer's frame of reference is assumed to fluctuate. We investigate the average energy and molar specific heat at constant volume of an Einstein solid measured by an observer with fluctuating frame of reference. The Einstein solid consists of $N$ identical non-interacting simple harmonic oscillators per mole, where $N$ is the Avogadro's number at temperature $T$. The average energy and molar specific heat at constant volume of the Einstein solid are formulated for two types of fluctuations of the observer's frame of reference in order to consider pedagogical and experimental demonstrations. The average energy of the Einstein solid is formulated from the definition of canonical ensemble average and the molar specific heat at constant volume of it is calculated by differentiating the average energy with $T$. The molar specific heat at constant volume of the Einstein solid exhibits novel features at low temperatures according to the distribution of fluctuations of the observer's frame of reference: 0 and $3 R$ at $T=0 \mathrm{~K}$ for square-wave and sawtooth-wave fluctuations, respectively, where $R$ is the gas constant.
\end{abstract}

Keywords: Einstein Solids, Specific Heat, Harmonic Oscillators, Fluctuations, Observer Effect, Thermodynamic Law

\section{Introduction}

The observer effect in physics is the theory that observing an object or phenomenon changes that phenomenon. This effect can be reduced to insignificance in performing a measurement in classical physics because the disturbance associated with the measurement can be made small with arbitrary precision [1]. The observer effect is thought to be inevitable in quantum mechanics because the measurement of an object involves an interaction with an observer or apparatus, which introduces an unavoidable random disturbance on the object [2]. This point of view is a fundamental aspect of the Copenhagen interpretation of quantum mechanics [3].

The Heisenberg uncertainty relation, a corner stone of the Copenhagen interpretation of quantum mechanics, states that any precise measurement of the position (momentum) of an object is allowed with momentum (position) disturbance, whereas simultaneous information about both position and momentum of an object is limited by $h / 4 \pi$ [4], where $h$ is the Planck's constant. The uncertainty relation has been refined by introducing the notion of standard deviation [5], and generalized to a pair of observables (for example, time and energy) [6]. Of the observer effect and the Heisenberg uncertainty relation, M. Ozawa and K. Okamura $[7,8]$ have said that throughout the history of quantum mechanics, the observer effect has often been confused with the wavenature-induced uncertainty principle.

Wave function collapse is a key issue regarding the theoretical foundation of the concept of measurement in quantum mechanics, where the wave function of an object is expressed as the superposition of all the eigenstates of the object prior to a measurement being performed. When a measurement is performed, the wave function of an object is 
collapsed to a single and definite value [9]. How the wave function of the object collapses is still on debate [10-19].

The observer effect in quantum mechanics has been studied under the assumption that an observer's frame of reference remains constant - conventionally, it is taken to be equal to zero - throughout the performance of a measurement. Thus, it is an unresolved problem as to what is actually measured throughout the performance of a measurement during which the observer's frame of reference is assumed to fluctuate. The observer effect induced by fluctuations of an observer's frame of reference is the principal issue in this study.

We investigate the average energy and molar specific heat at constant volume of an Einstein solid measured by an observer with fluctuating frame of reference. We formulate the average energy and molar specific heat of the Einstein solid for two types of fluctuations of the observer's frame of reference in order to consider pedagogical and experimental demonstrations: square-wave and sawtooth-wave fluctuations, in time representations. The molar specific heat at constant volume of the Einstein solid exhibits novel features at low temperatures: it is 0 at temperature $T=0 \mathrm{~K}$ but it has a peak at low temperatures for square-wave fluctuations; and it converges to $3 R$ for sawtooth-wave fluctuations where $R$ is the gas constant. In this regard, the molar specific heat at constant volume of the Einstein solid with a varying temperature would reveal the distribution of fluctuations of the observer's frame of reference.

\section{The Average Energy of a Simple Harmonic Oscillator}

A one-dimensional (1D) quantum-mechanical simple harmonic oscillator (QSHO) [20] is an element of an Einstein solid, the Hamiltonian, $H$, of which is expressed as $H=$ $\frac{1}{2 m_{0}} p^{2}+\frac{1}{2} m_{0} \omega_{0}^{2} x^{2}$, where $p$ and $x$ are the momentum and position operators, respectively; $m_{0}$ is the mass of the oscillator; and $\omega_{0}(=2 \pi f)$ is the angular frequency. The energy of the nth eigenstate $E_{n}$ is expressed as $E_{n}=$ $\left(n+\frac{1}{2}\right) h f$, where $n=0,1,2, \cdots$. Given an observer with fluctuating frame of reference, $E_{r e f}(t)$, the measured energy for $E_{n}$ at time $t, E_{n}(t)$, can be expressed as $E_{n}(t)=$ $E_{n}-E_{\text {ref }}(t)$ (see figure 1 ).

The average energy of the 1D QSHO with time interval $\Delta \mathrm{t}\left(=t_{f}-t_{i}\right)$ under $E_{r e f}(t), u$, is expressed as

(a)

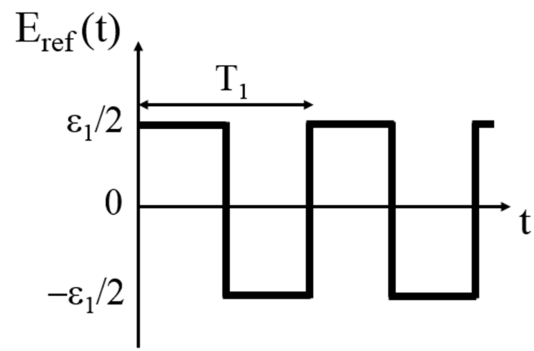

$$
u=\frac{\sum_{n=0}^{\infty} \int_{t_{i}}^{t_{f}} E_{n}(t) \exp \left(-\beta E_{n}(t)\right) d t}{\sum_{n=0}^{\infty} \int_{t_{i}}^{t_{f}} \exp \left(-\beta E_{n}(t)\right) d t},
$$

where $t_{i}$ and $t_{f}$ are the initial and final times measured by the observer, respectively, and $\beta=1 / k T$, where $k$ is the Boltzmann's constant.

An Einstein solid consists of $N$ identical non-interacting 3D QSHOs per mole, where $N$ is the Avogadro's number. The average energy of the Einstein solid, $U$, is expressed as

$$
U=3 N u \text {. }
$$

(a)

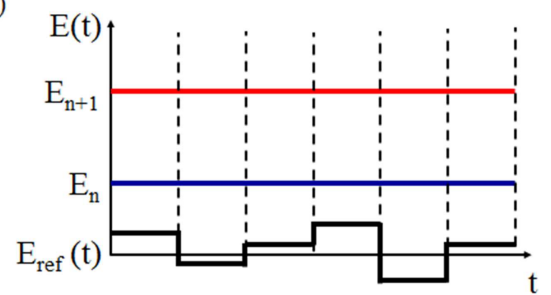

(b)

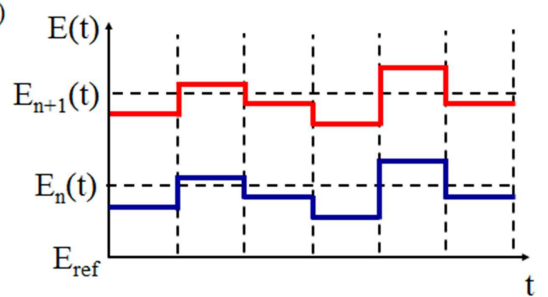

Figure 1. Schematic energy diagram of a quantum-mechanical simple harmonic oscillator (QSHO). (a) The energy of the nth eigenstate, $E_{n}$, where $n=0,1,2, \cdots$. Fluctuating frame of reference at time $t, E_{\text {ref }}(t)$. (b) Measured energy for $E_{n}$ at time $t, E_{n}(t)\left(=E_{n}-E_{\text {ref }}(t)\right)$. Stationary frame of reference, $E_{\text {ref }}$, is taken to be equal to 0 .

\section{An Einstein Solid under Square-Wave Fluctuations}

Given periodic $\mathrm{E}_{\text {ref }}(t)=\left\{\begin{array}{c}\varepsilon_{1} / 2 \text { for } 0 \leq t<T_{1} / 2 \\ -\varepsilon_{1} / 2 \text { for } T_{1} / 2 \leq t<T_{1}\end{array}\right.$ (see figure 2 (a)), the measured energy for $E_{n}$ at time $t$, $E_{n}(t)$, can be expressed as

$$
\mathrm{E}_{n}(t)=\left\{\begin{array}{l}
\mathrm{E}_{n}-\varepsilon_{1} / 2 \text { for } 0 \leq t<T_{1} / 2 \\
\mathrm{E}_{n}+\varepsilon_{1} / 2 \text { for } T_{1} / 2 \leq t<T_{1}
\end{array},\right.
$$

where $\varepsilon_{1} / 2$ and $T_{1}$ are the amplitude and period of the square-wave fluctuations.

(b)

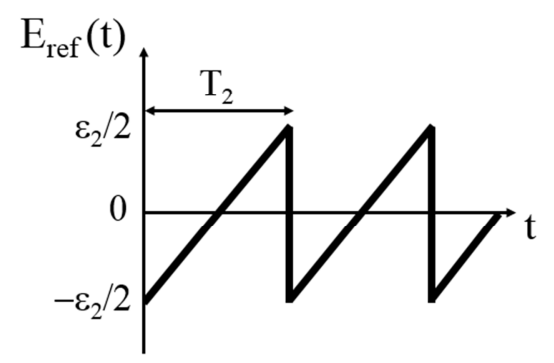

Figure 2. (a) Fluctuating frame of reference at time $t, E_{\text {ref }}(t)$, for square-wave fluctuations, whose amplitude and period are $\varepsilon_{1} / 2$ and $T_{1}$, respectively. (b) Fluctuating frame of reference at time $t, E_{\text {ref }}(t)$, for sawtooth-wave fluctuations, whose amplitude and period are $\varepsilon_{2} / 2$ and $T_{2}$, respectively. 


\subsection{Square-Wave Fluctuations with a Half Period}

For an observer with fluctuating frame of reference by means of half-period square-wave fluctuations, the corresponding average energy of the Einstein solid, $U_{0}$, is expressed as

$$
U_{0}=3 N \frac{\sum_{n=0}^{\infty}\left(E_{n}-\varepsilon_{1} / 2\right) \exp \left(-\beta E_{n}+\beta \varepsilon_{1} / 2\right)}{\sum_{n=0}^{\infty} \exp \left(-\beta E_{n}+\beta \varepsilon_{1} / 2\right)}=3 N \frac{h f}{2} \operatorname{coth}\left(\frac{h f}{2 k T}\right)-3 N \frac{\varepsilon_{1}}{2},
$$

where $t_{i}=0$ and $t_{f}=T_{1} / 2$.

$$
U_{0}=3 N \frac{\sum_{n=0}^{\infty}\left(E_{n}+\varepsilon_{1} / 2\right) \exp \left(-\beta E_{n}-\beta \varepsilon_{1} / 2\right)}{\sum_{n=0}^{\infty} \exp \left(-\beta E_{n}-\beta \varepsilon_{1} / 2\right)}=3 N \frac{h f}{2} \operatorname{coth}\left(\frac{h f}{2 k T}\right)+3 N \frac{\varepsilon_{1}}{2},
$$

where $t_{i}=T_{1} / 2$ and $t_{f}=T_{1}$.

The molar specific heat at constant volume of the Einstein solid under half-period square-wave fluctuations of the observer's frame of reference, $C_{v 0}$, is expressed as

$$
C_{v 0}=\left(\frac{d U_{0}}{d T}\right)_{v}=3 R \frac{\left(\frac{\theta_{E}}{2 T}\right)^{2}}{\sinh ^{2}\left(\frac{\theta_{E}}{2 T}\right)},
$$

where $\theta_{E}=h f / k$ is an Einstein temperature and $R=N k$. For all $\theta_{E}>0, C_{v 0}$ is 0 at $T=0 \mathrm{~K}$, monotonically increases with $T$, and saturates to $3 R[21]$ at high-temperature limits $\left(T / \theta_{E} \gg 1\right)$, which agrees with that of the Einstein solid published in 1906 [22].

\subsection{Square-Wave Fluctuations at Low-Frequency Limits}

For an observer with fluctuating frame of reference by means of square-wave fluctuations at low-frequency limits, the corresponding average energy of the Einstein solid, $U_{0}{ }^{\prime}$, is expressed as

$$
U_{0}{ }^{\prime}=3 N \frac{\sum_{n=0}^{\infty}\left(E_{n}-\varepsilon_{1} / 2\right) \exp \left(-\beta E_{n}+\beta \varepsilon_{1} / 2\right)}{\sum_{n=0}^{\infty} \exp \left(-\beta E_{n}+\beta \varepsilon_{1} / 2\right)}=3 N \frac{h f}{2} \operatorname{coth}\left(\frac{h f}{2 k T}\right)-3 N \frac{\varepsilon_{1}}{2},
$$

where $t_{i}=0$ and $t_{f} \ll T_{1} / 2$.

$$
U_{0}^{\prime}=3 N \frac{\sum_{n=0}^{\infty}\left(E_{n}+\varepsilon_{1} / 2\right) \exp \left(-\beta E_{n}-\beta \varepsilon_{1} / 2\right)}{\sum_{n=0}^{\infty} \exp \left(-\beta E_{n}-\beta \varepsilon_{1} / 2\right)}=3 N \frac{h f}{2} \operatorname{coth}\left(\frac{h f}{2 k T}\right)+3 N \frac{\varepsilon_{1}}{2},
$$

where $t_{i}=T_{1} / 2$ and $t_{f} \ll T_{1}$.

The molar specific heat at constant volume of the Einstein solid under square-wave fluctuations of the observer's frame of reference at low-frequency limits, $C_{v 0}{ }^{\prime}$, is expressed as

$$
C_{v 0}^{\prime}=\left(\frac{d U_{0}^{\prime}}{d T}\right)_{v}=C_{v 0}
$$

\subsection{Square-Wave Fluctuations with One Period}

For an observer with fluctuating frame of reference by means of one-period square-wave fluctuations, the corresponding average energy of the Einstein solid, $U_{1}$, is expressed as

$$
\begin{gathered}
U_{1}=3 N \frac{\sum_{n=0}^{\infty}\left\{\left(E_{n}-\varepsilon_{1} / 2\right) \exp \left(-\beta E_{n}+\beta \varepsilon_{1} / 2\right)+\left(E_{n}+\varepsilon_{1} / 2\right) \exp \left(-\beta E_{n}-\beta \varepsilon_{1} / 2\right)\right\}}{\sum_{n=0}^{\infty}\left\{\exp \left(-\beta E_{n}+\beta \varepsilon_{1} / 2\right)+\exp \left(-\beta E_{n}-\beta \varepsilon_{1} / 2\right)\right\}} \\
=3 N \frac{h f}{2} \operatorname{coth}\left(\frac{h f}{2 k T}\right)-3 N \frac{\varepsilon_{1}}{2} \tanh \left(\frac{\varepsilon_{1}}{2 k T}\right),
\end{gathered}
$$

$t_{i}=0$ and $t_{f}=T_{1}$.

The molar specific heat at constant volume of the Einstein solid under one-period square-wave fluctuations of the observer's frame of reference, $C_{v 1}$, is expressed as

$$
C_{v 1}=\left(\frac{d U_{1}}{d T}\right)_{v}=3 R \frac{\left(\frac{\theta_{E}}{2 T}\right)^{2}}{\sinh ^{2}\left(\frac{\theta_{E}}{2 T}\right)}+3 R \frac{\left(\frac{\theta_{I}}{2 T}\right)^{2}}{\cosh ^{2}\left(\frac{\theta_{I}}{2 T}\right)}
$$

where $\theta_{I}=\varepsilon_{1} / k$. 


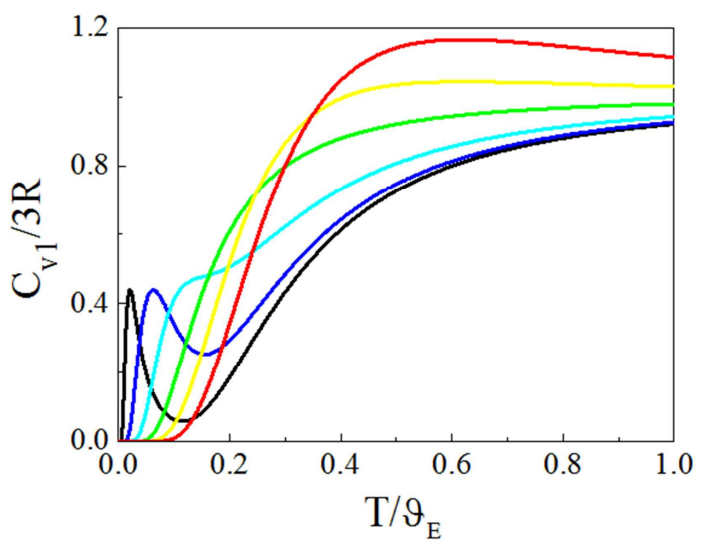

Figure 3. Molar specific heat at constant volume divided by $3 R, C_{v 1} / 3 R$, as a function of $T / \theta_{E}$ for various $\theta_{I} / \theta_{E}$, where $R$ is the gas constant. Here, $\theta_{I} / \theta_{E}=0.99$ (red), 0.7 (yellow), 0.5 (green), 0.3 (cyan), 0.15 (blue), and 0.05 (black).
In figure $3, C_{v 1} / 3 R$ is displayed as a function of $T / \theta_{E}$ for various $\theta_{I} / \theta_{E}$. The molar specific heat at constant volume of the Einstein solid under one-period square-wave fluctuations of the observer's frame of reference $\left(C_{v 1}\right)$ is 0 at $T=0 \mathrm{~K}$, monotonically increases with $T$, and saturates to $3 R$ [21] at high-temperature limits $\left(T / \theta_{E} \gg 1\right)$. However, it has a peak at $T / \theta_{E} \approx 0.02$ for $\theta_{I} / \theta_{E}=0.05$, which, induced by oneperiod square-wave fluctuations of the observer's frame of reference, is gradually broadened with $\theta_{I} / \theta_{E}$.

\subsection{Square-Wave Fluctuations at High-Frequency Limits}

For an observer with fluctuating frame of reference by means of square-wave fluctuations at high-frequency limits, the corresponding average energy of the Einstein solid, $U_{1}{ }^{\prime}$, is expressed as

$$
U_{1}^{\prime} \approx 3 N \frac{\sum_{n=0}^{\infty}\left\{\left(E_{n}-\varepsilon_{1} / 2\right) \exp \left(-\beta E_{n}+\beta \varepsilon_{1} / 2\right)+\left(E_{n}+\varepsilon_{1} / 2\right) \exp \left(-\beta E_{n}-\beta \varepsilon_{1} / 2\right)\right\}}{\sum_{n=0}^{\infty}\left\{\exp \left(-\beta E_{n}+\beta \varepsilon_{1} / 2\right)+\exp \left(-\beta E_{n}-\beta \varepsilon_{1} / 2\right)\right\}}=U_{1}
$$

where $t_{i}=0$ and $t_{f} \gg T_{1}$.

The molar specific heat at constant volume of the Einstein solid under square-wave fluctuations of the observer's frame of reference at high-frequency limits, $C_{v 1}{ }^{\prime}$, is expressed as

$$
C_{v 1}^{\prime}=\left(\frac{d U_{1}^{\prime}}{d T}\right)_{v} \approx C_{v 1}
$$

\section{An Einstein Solid under Sawtooth-Wave Fluctuations}

Given periodic $\mathrm{E}_{\text {ref }}(t)=\frac{\varepsilon_{2}}{T_{2}} t-\frac{\varepsilon_{2}}{2}$ for $0 \leq t<T_{2}$ (see figure $2(\mathrm{~b})$ ), the measured energy for $E_{n}$ at time $t, E_{n}(t)$, can be expressed as

$$
\mathrm{E}_{n}(t)=E_{n}-\frac{\varepsilon_{2}}{T_{2}} t+\frac{\varepsilon_{2}}{2}, \text { for } 0 \leq t<T_{2},
$$

where $\varepsilon_{2} / 2$ and $T_{2}$ are the amplitude and period of the sawtooth-wave fluctuations.

\subsection{Sawtooth-Wave Fluctuations with One Period}

For an observer with fluctuating frame of reference by means of one-period sawtooth-wave fluctuations, the corresponding average energy of the Einstein solid, $U_{2}$, is expressed as

$$
\begin{array}{r}
U_{2}=3 N \frac{\sum_{n=0}^{\infty} \int_{0}^{T_{2}} E_{n}(t) \exp \left(-\beta E_{n}(t)\right) d t}{\sum_{n=0}^{\infty} \int_{0}^{T_{2}} \exp \left(-\beta E_{n}(t)\right) d t} \\
=3 N \frac{h f}{2} \operatorname{coth}\left(\frac{h f}{2 k T}\right)+3 N k T\left\{1-\frac{\varepsilon_{2}}{2 k T} \operatorname{coth}\left(\frac{\varepsilon_{2}}{2 k T}\right)\right\} .
\end{array}
$$

The molar specific heat at constant volume of the Einstein solid under one-period sawtooth-wave fluctuations of the observer's frame of reference, $C_{v 2}$, is expressed as

$$
C_{v 2}=\left(\frac{d U_{2}}{d T}\right)_{v}=3 R \frac{\left(\frac{\theta_{E}}{2 T}\right)^{2}}{\sinh ^{2}\left(\frac{\theta_{E}}{2 T}\right)}+3 R\left\{1-\frac{\left(\frac{\theta_{S}}{2 T}\right)^{2}}{\sinh ^{2}\left(\frac{\theta_{S}}{2 T}\right)}\right\},
$$

where $\theta_{S}=\varepsilon_{2} / k$.

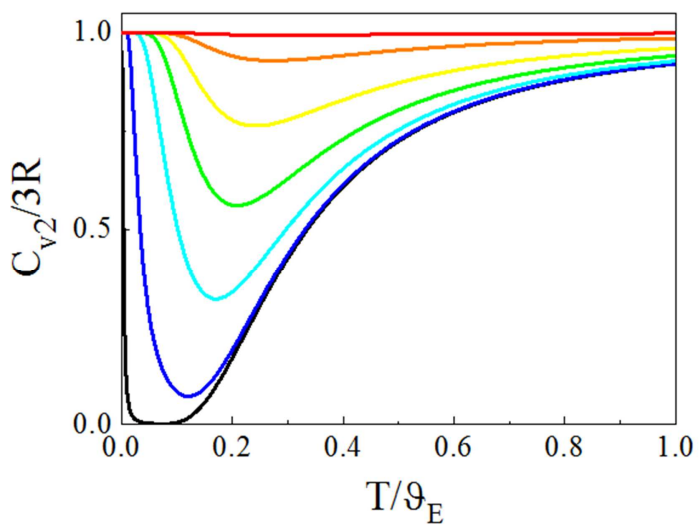

Figure 4. Molar specific heat at constant volume divided by $3 R, C_{v 2} / 3 R$, as a function of $T / \theta_{E}$ for various $\theta_{S} / \theta_{E}$, where $\theta_{S} / \theta_{E}=0.99$ (red), 0.9 (orange), 0.7 (yellow), 0.5 (green), 0.3 (cyan), 0.1 (blue), and 0.01 (black).

In figure $4, C_{v 2} / 3 R$ is displayed as a function of $T / \theta_{E}$ for various $\theta_{S} / \theta_{E}$. The molar specific heat at constant volume of the Einstein solid under one-period sawtooth-wave fluctuations of the observer's frame of reference $\left(C_{v 2}\right)$ is $3 R$ at $T=0 \mathrm{~K}$, monotonically decreases, reaches a minimum, monotonically increases with $T$, and saturates to $3 R$ [21] at high-temperature limits $\left(T / \theta_{E} \gg 1\right)$. The minimum increases with $\theta_{S} / \theta_{E}$ and saturates to $3 R$. The one-period sawtoothwave fluctuations induce the convergence of $C_{v 2}=3 R$ at $T$ $=0 \mathrm{~K}-\mathrm{a}$ phenomenon that is not governed by the third thermodynamic law [23, 24], which states that the specific heat of any Einstein solid is 0 at $T=0 \mathrm{~K}$, but governed by the Dulong-Petit law [21].

\subsection{Sawtooth-Wave Fluctuations at Low-Frequency Limits}

For an observer with fluctuating frame of reference by 
means of sawtooth-wave fluctuations at low-frequency limits, the corresponding average energy of the Einstein solid, $U_{2}{ }^{\prime}$, is expressed as

$$
\begin{gathered}
U_{2}^{\prime} \approx 3 N \frac{\sum_{n=0}^{\infty}\left(E_{n}+\alpha\right) \exp \left(-\beta E_{n}-\beta \alpha\right)}{\sum_{n=0}^{\infty} \exp \left(-\beta E_{n}-\beta \alpha\right)}= \\
3 N \frac{h f}{2} \operatorname{coth}\left(\frac{h f}{2 k T}\right)+3 N \alpha,
\end{gathered}
$$

where $0<\Delta \mathrm{t} \ll T_{2}$ and $-\varepsilon_{2} / 2 \leq \alpha \leq \varepsilon_{2} / 2$.

The molar specific heat at constant volume of the Einstein solid under sawtooth-wave fluctuations of the observer's frame of reference at low-frequency limits, $C_{v 2}{ }^{\prime}$, is expressed as

$$
C_{v 2}^{\prime}=\left(\frac{d U_{2}^{\prime}}{d T}\right)_{v} \approx C_{v 0}
$$

\subsection{Sawtooth-Wave Fluctuations at High-Frequency Limits}

For an observer with fluctuating frame of reference by means of sawtooth-wave fluctuations at high-frequency limits, the corresponding average energy of the Einstein solid, $U_{2}{ }^{\prime \prime}$, is expressed as

$$
U_{2}^{\prime \prime} \approx 3 N \frac{\sum_{n=0}^{\infty} \int_{0}^{T_{2}} E_{n}(t) \exp \left(-\beta E_{n}(t)\right) d t}{\sum_{n=0}^{\infty} \int_{0}^{T_{2}} \exp \left(-\beta E_{n}(t)\right) d t}=U_{2},
$$

where $\Delta \mathrm{t} \gg T_{2}$.

The molar specific heat at constant volume of the Einstein solid under sawtooth-wave fluctuations of the observer's frame of reference at high-frequency limits, $C_{v 2}{ }^{\prime \prime}$, is expressed as

$$
C_{v 2}^{\prime \prime}=\left(\frac{d U_{2}^{\prime \prime}}{d T}\right)_{v} \approx C_{v 2}
$$

\section{Conclusions}

In this paper, we succeeded in introducing a novel observer effect in an Einstein solid under fluctuations of an observer's frame of reference. The average energy and corresponding molar specific heat at constant volume of the Einstein solid are formulated for two types of fluctuations of the observer's frame of reference: periodic square-wave and sawtooth-wave fluctuations, in time representations. Under the square-wave fluctuations with one period or at highfrequency limits, the molar specific heat at constant volume of the Einstein solid at high-temperature limits is similar with that of a conventional Einstein solid. However, it has a peak at low temperatures under the fluctuations and is gradually broadened with the amplitude of the fluctuations. Under the sawtooth-wave fluctuations with one period or at highfrequency limits, the molar specific heat at constant volume of the Einstein solid at high-temperature limits is also similar with that of the conventional Einstein solid. However, it is $3 R$ at $T=0 \mathrm{~K}$, monotonically decreases, reaches a minimum, and monotonically increases with $T$ at low temperatures. Therefore, the molar specific heat at constant volume of the Einstein solid at low temperatures would provide criteria for characterizing the distribution of fluctuations of the observer's frame of reference.

\section{Acknowledgements}

This work was supported by Sejong Academy of Science and Arts.

\section{References}

[1] A. C. Phillips, Introduction to Quantum Mechanics, John Wiley \& Sons Ltd., 2003, pp. 10-17.

[2] M. Ozawa, "Physical content of Heisenberg's uncertainty relation: limitation and reformulation," Phys. Lett. A, vol. 318, pp. 21-29, 2003.

[3] N. Bohr, "The quantum postulate and the recent development of atomic theory," Nature, vol. 121, pp. 580-590, 1928.

[4] W. Heisenberg, "Über den anschaulichen Inhalt der quantentheoretischen Kinematik und Mechanik," Z. Physik, vol. 43, pp. 172-198, 1927.

[5] E. H. Kennard, "Zur Quantenmechanik einfacher Bewegungstypen,” Z. Physik, vol. 44, pp. 326-352, 1927.

[6] H. P. Robertson, "The uncertainty principle," Phys. Rev., vol. 34, pp. 163-164, 1929.

[7] M. Ozawa, "Universally valid reformulation of the Heisenberg uncertainty principle on noise and disturbance in measurement," Phys. Rev. A, vol. 67, pp. 042105, 2003.

[8] K. Okamura, M. Ozawa, "Universally valid uncertainty relations in general quantum systems," arXiv: 1808. 10615v2, pp. 1-16, 2018.

[9] R. Penrose, The road to reality: a complete guide to the laws of the universe, Jonathan Cape, 2004, pp. 516-517.

[10] S. Weinberg, The Oxford History of the Twentieth Century, Oxford University Press, 1998, pp. 22-34.

[11] S. Weinberg, "Einstein's Mistakes," Physics Today, pp. 31-35, 2005.

[12] W. H. Zurek, "Decoherence, einselection, and the quantum origins of the classical,” Rev. Mod. Phys., vol. 75, pp. 715-775, 2003.

[13] E. Joos and H. D. Zeh, "The emergence of classical properties through interaction with the environment," Z. Phys. B, vol. 59, vol. 223-243, 1985.

[14] H. D. Zeh, E. Joos, H. D. Zeh, C. Kiefer, D. Giulini, J. Kupsch, and I.-O. Stamatescu, Decoherence and the Appearance of a Classical World in Quantum Theory, 2nd ed., Springer-Verlag, 2003, pp. 16-26.

[15] V. P. Belavkin, "Nondemolition principle of quantum measurement theory," Foundations of Physics, vol. 24, pp. 685-714, 1994.

[16] V. P. Belavkin, "Quantum noise, bits and jumps: uncertainties, decoherence, measurements and filtering," Progress in Quantum Electronics, vol. 25, pp. 1-53, 2001.

[17] M. Schlosshauer, "Decoherence, the measurement problem, and interpretations of quantum mechanics," Rev. Mod. Phys., vol. 76, pp. 1267, 2005. 
[18] M. Schlosshauer, "Experimental motivation and empirical consistency in minimal no-collapse quantum mechanics," Ann. Phys., vol. 321, pp. 112-149, 2006.

[19] M. Schlosshauer, "Measuring the quantum state of a single system with minimum state disturbance," Phys. Rev. A, vol. 93, pp. 012115, 2016.

[20] E. Schrödinger, "Der stetige Übergang von der Mikro- zur Makromechanik," Die Naturwissenschaften, vol. 14, pp. 664666, 1926.

[21] A. T. Petit and P. L. Dulong, "Recherches sur quelques points importants de la Théorie de la Chaleur," Annales de Chimie et de Physique, vol. 10, pp. 395-413, 1819.
[22] A. Einstein, "Die Plancksche Theorie der Strahlung und die Theorie der spezifischen Wärme," Ann. d. Physik, vol. 22, pp. 180-190, 1907.

[23] J. Wilks, The Third Law of Thermodynamics, Oxford University Press, 1961, pp. 32-36.

[24] C. Kittel and H, Kroemer, Thermal Physics, 2nd ed., W. H. Freeman, San Francisco, 1980, pp. 48-49. 